\title{
Avaliação do vigor de sementes de milho superdoce por meio da análise computadorizada de imagens de plântulas ${ }^{1}$
}

\author{
Renata Oliveira Alvarenga ${ }^{2 *}$, Julio Marcos-Filho², Francisco Guilhien Gomes Junior ${ }^{2}$
}

\begin{abstract}
RESUMO - O presente trabalho foi conduzido com o objetivo de verificar a eficiência do sistema computadorizado de avaliação do vigor de sementes (SVIS ${ }^{\circledR}$ ), em comparação com outros procedimentos disponíveis, em sementes de milho superdoce. Foram utilizadas sementes dos híbridos SWB 551 e SWB 585, cada uma representada por cinco lotes, conduzindo-se testes de germinação, envelhecimento acelerado (tradicional e com solução saturada de $\mathrm{NaCl}$ ) e emergência de plântulas em campo. Para a determinação do vigor das sementes por meio do SVIS ${ }^{\circledR}$, as plântulas foram avaliadas aos três dias após a semeadura do teste de germinação, sendo obtidos dados referentes ao índice de vigor, à uniformidade de desenvolvimento e ao comprimento de plântulas. Para ambos os híbridos avaliados, os resultados fornecidos pelo SVIS ${ }^{\circledR}$ foram coerentes com os do teste de envelhecimento acelerado tradicional. Concluiu-se que o SVIS ${ }^{\circledR}$ é eficiente para avaliação do vigor de sementes de milho superdoce, constituindo alternativa viável para a determinação do potencial fisiológico.
\end{abstract}

Termos para indexação: Zea mays L., potencial fisiológico, análise de sementes.

\section{Evaluation of supersweet corn seed vigor using an automated computer imaging system}

\begin{abstract}
The objective of this study was to verify the efficiency of an automated system for seedling evaluation $\left(\right.$ SVIS $\left.^{\circledR}\right)$ to assess supersweet corn seed vigor compared to information from accepted vigor tests. Five seed lots, each of the SWB 551 and SWB 585 hybrids, were subjected to germination, accelerated aging (traditional and saturated salt) and field seedling emergence tests. The Seed Vigor Imaging System (SVIS ${ }^{\circledR}$ ) parameters comprised mean seedling growth, uniformity and a vigor index, computed in scanned images of three-day old seedlings from the germination test. The results for both hybrids provided by SVIS ${ }^{\circledR}$ were comparable to those from the traditional accelerated aging test, thus demonstrating an efficient vigor evaluation of supersweet corn seeds.
\end{abstract}

Index terms: Zea mays L., physiological potential, seed analysis.

\section{Introdução}

A utilização de sementes de alta qualidade é imprescindível para o estabelecimento adequado da cultura do milho superdoce. No entanto, as sementes desse tipo de milho apresentam características que contribuem para seu baixo potencial fisiológico como, por exemplo, o baixo teor de amido, a alta quantidade de açúcares no endosperma, a alta sensibilidade aos danos durante a embebição, a fragilidade do sistema de membranas após a secagem e a sensibilidade à ação de fungos patogênicos (Tosello, 1987; Chern e Sung, 1991; Douglas et al., 1993; Silva, 1994). Dessa maneira, a avaliação eficiente da germinação e do vigor de sementes constitui etapa importante do processo de produção de sementes de milho superdoce.

Visando complementar as informações obtidas no teste

${ }^{1}$ Submetido em 30/09/2011. Aceito para publicação em 19/12/2011.

${ }^{2}$ Departamento de Produção Vegetal, USP/ESALQ, Caixa Postal 9, 13418-900-Piracicaba, SP, Brasil.

*Autor para correspondência <realvarenga7@gmail.com> 
de germinação, os testes de vigor vêm sendo cada vez mais utilizados. As empresas produtoras têm incluído esses testes em programas internos de controle para garantir a qualidade das sementes destinadas à comercialização. Vários testes têm sido conduzidos para a determinação do vigor de sementes de milho superdoce, destacando-se os de envelhecimento acelerado, frio, condutividade elétrica, primeira contagem de germinação e emergência de plântulas em campo (Guiscem et al., 2002; Santos et al., 2002; Ramos et al., 2008).

Um dos métodos mais importantes para a avaliação do vigor de sementes consiste na determinação da velocidade e uniformidade de crescimento (Hoffmaster et al., 2003). Assim, os testes que avaliam o crescimento de plântulas são sugeridos pelas duas principais associações internacionais que reúnem tecnologistas de sementes (ISTA - International Seed Testing Association e AOSA - Association of Official Seed Analysts), apresentando eficiência na avaliação do potencial fisiológico de sementes de várias espécies, dentre elas, o milho doce (Wann, 1980; Styer et al., 1980; Styer e Cantliffe, 1984). Contudo, esses testes podem consumir período de tempo relativamente amplo e produzir resultados variáveis entre laboratórios e analistas. Diante disso, o uso da análise computadorizada de imagens constitui uma das alternativas inovadoras, favorecendo tanto a redução do período necessário para a condução do teste, como a consistência das informações obtidas.

Com o objetivo de avaliar o vigor de sementes, McCormac et al. (1990) talvez tenham sido os primeiros a utilizar a análise computadorizada de imagens, determinando o comprimento da raiz primária de plântulas de alface e cenoura. Posteriormente, Sako et al. (2001) desenvolveram um sistema automatizado para avaliação do vigor de sementes de alface, denominado Seed Vigor Imaging System $\left(\right.$ SVIS $^{\circledR}$ ), o qual gera valores referentes ao índice de vigor (valores de 0 a 1000 , diretamente proporcionais ao vigor), uniformidade de desenvolvimento (também de 0 a 1000) e comprimento de plântulas. Este sistema também tem demonstrado eficiência na avaliação do potencial fisiológico de sementes de outras espécies, como soja (Hoffmaster et al., 2003; Hoffmaster et al., 2005; Marcos-Filho et al., 2009), milho (Hoffmaster et al., 2005; Otoni e McDonald, 2005), melão (Marcos-Filho et al., 2006) e feijão (Gomes Junior e Chamma, 2008), sendo sua utilização estendida para a avaliação da eficiência do condicionamento fisiológico de sementes de milho doce (Gomes Junior et al., 2009).

O procedimento para avaliação do vigor de sementes por meio do $\mathrm{SVIS}^{\circledR}$ é simples e rápido. O tempo necessário para gerar um índice de vigor a partir de imagens de plântulas com três dias de idade é menor que o requerido para executar o teste manualmente (Hoffmaster et al., 2005). De acordo com Gomes Junior et al. (2009), os resultados para uma amostra de 50 sementes podem ser obtidos de dois a três minutos. Outras vantagens desse sistema incluem a eliminação do erro humano, aumentando a confiabilidade dos dados para fins de comparação e a possibilidade de arquivamento das imagens para análise posterior.

Diante disso, a presente pesquisa foi conduzida com o objetivo de avaliar a eficiência do sistema computadorizado de análise de imagens de plântulas (SVIS ${ }^{\circledR}$ ) para avaliação do vigor de sementes de milho superdoce, em comparação com procedimentos comumente utilizados em sementes dessa espécie.

\section{Material e Métodos}

A pesquisa foi conduzida nos Laboratórios de Análise de Sementes e de Análise de Imagens do Departamento de Produção Vegetal da Escola Superior de Agricultura "Luiz de Queiroz", Universidade de São Paulo (USP/ESALQ), em Piracicaba/SP.

Utilizaram-se dois híbridos de milho superdoce $(\operatorname{sh} 2)$, SWB 551 e SWB 585, fornecidos pela empresa Dow AgroSciences, cada um representado por cinco lotes de sementes tratadas com fungicida e inseticida. Para obtenção de lotes com potenciais fisiológicos distintos, parte de cada amostra de sementes recebida foi submetida ao envelhecimento artificial adotando-se os seguintes procedimentos: para o híbrido SWB 551 foi recebido um lote de sementes com $8,7 \mathrm{~kg}$ (lote 1). Esse lote foi dividido em quatro partes; três delas foram envelhecidas artificialmente a $41{ }^{\circ} \mathrm{C}$ durante 24,48 e 72 horas, constituindo os lotes 2,3 e 4 , respectivamente. O quinto lote (lote 5) foi obtido mediante a mistura de 250,0 g do lote 1 com 170,0 g do lote 2, com base em resultados de testes preliminares para avaliação do potencial fisiológico. O procedimento para a obtenção dos lotes do híbrido SWB 585 foi semelhante, sendo o lote 6 correspondente às sementes não envelhecidas artificialmente e, os lotes 7 , 8 e 9 , às sementes envelhecidas a $41^{\circ} \mathrm{C}$, durante $12,24 \mathrm{e}$ 48 horas, respectivamente; o lote 10 foi obtido mediante a mistura de 408,0 g do lote 6 com 175,0 g do lote 8 .

Após o preparo e cuidadosa homogeneização dos lotes de cada híbrido, as sementes foram colocadas para secar, a temperatura ambiente (aproximadamente $25^{\circ} \mathrm{C}$ ), até atingirem teores de água próximos aos iniciais. Em seguida, as sementes de cada lote foram colocadas em sacos 
de papel e armazenadas em câmara seca $\left(20{ }^{\circ} \mathrm{C}\right.$ e $50 \%$ de umidade relativa do ar), onde permaneceram durante todo o período experimental.

As sementes do híbrido SWB 551 foram avaliadas em três épocas (com intervalos trimestrais) e, as do SWB 585, em duas épocas experimentais (com intervalo semestral), sendo submetidas aos seguintes procedimentos: determinação do grau de umidade - efetuada antes da instalação dos testes de germinação e vigor, pelo método da estufa a $105^{\circ} \mathrm{C}\left( \pm 3{ }^{\circ} \mathrm{C}\right)$, durante 24 horas (Brasil, 2009), com duas repetições para cada lote. Os resultados foram expressos em porcentagem média (base úmida) por lote; germinação - conduzido com quatro repetições de 50 sementes por lote, em rolos de papel-toalha umedecidos com quantidade de água equivalente a 2,5 vezes a massa do papel seco, a $25{ }^{\circ} \mathrm{C}$, por sete dias. As avaliações foram efetuadas de acordo com os critérios estabelecidos nas Regras para Análise de Sementes (Brasil, 2009). Os resultados foram expressos em porcentagem média de plântulas normais para cada lote; envelhecimento acelerado (procedimento tradicional) - conduzido em caixas de plástico $(11,0 \times 11,0 \times 3,0 \mathrm{~cm})$ possuindo em seu interior suportes para apoio de uma tela metálica, onde as sementes foram distribuídas de maneira a formar uma camada única. No interior de cada caixa foram adicionados $40 \mathrm{~mL}$ de água. As caixas foram tampadas e mantidas em câmara de envelhecimento (tipo BOD) regulada a $41{ }^{\circ} \mathrm{C}$, durante 72 horas (Hampton e TeKrony, 1995). Após esse período, foi conduzido o teste de germinação conforme descrito anteriormente, sendo a avaliação realizada aos quatro dias após a semeadura. Foi determinado, também, o grau de umidade das sementes após o período de envelhecimento, visando avaliação da uniformidade das condições do teste; envelhecimento acelerado com solução saturada de $\mathrm{NaCl}$ - foi conduzido conforme procedimento descrito para o teste de envelhecimento acelerado tradicional; porém, substituiu-se o volume de água adicionado às caixas por $40 \mathrm{~mL}$ de solução saturada de $\mathrm{NaCl}$, visando proporcionar ambiente com $76 \%$ de umidade relativa, de acordo com metodologia proposta por Jianhua e McDonald (1996). Após o envelhecimento, foi determinado o grau de umidade das sementes; emergência de plântulas em campo conduzida com quatro repetições de 50 sementes, cada uma delas representada por uma linha de $4 \mathrm{~m}$ de comprimento, espaçadas de $40 \mathrm{~cm}$, onde as sementes foram distribuídas de maneira equidistante, em sulcos com cerca de $7 \mathrm{~cm}$ de profundidade e cobertas com, aproximadamente, 3 $\mathrm{cm}$ de terra. Aos 14 dias após a semeadura foi avaliada a emergência de plântulas, registrando-se a porcentagem média de plântulas normais emersas para cada lote; análise computadorizada de imagens - SVIS®- as plântulas foram obtidas de quatro repetições de 20 sementes para cada lote, colocadas para germinar em duas fileiras situadas no terço superior do papel-toalha, a $25{ }^{\circ} \mathrm{C}$, durante três dias. Após esse período, as imagens foram captadas em scanner HP Scanjet $2410^{\circledR}$, disposto de maneira invertida no interior de uma caixa de alumínio $(60,0 \times 50,0 \times 12,0 \mathrm{~cm})$ e operado por software Photosmart ${ }^{\circledR}$, com resolução de 100 dpi. As plântulas normais identificadas em cada repetição foram transferidas do papel-toalha para uma folha de cartolina de coloração preta e colocada sobre a plataforma interna da caixa metálica. As imagens escaneadas foram analisadas pelo software Seed Vigor Imaging System $\left(\right.$ SVIS $\left.^{\circledR}\right)$, sendo obtidos valores médios do índice de vigor, uniformidade de desenvolvimento e comprimento de plântulas para cada lote e cultivar (Hoffmaster et al., 2005). Procedimento estatístico: Os dados obtidos foram submetidos à análise da variância, utilizando-se o software SAS ${ }^{\circledR}$ (SAS Institute, 2000). Foram utilizados os delineamentos inteiramente casualizado (testes em laboratório) e blocos ao acaso (teste em campo). As médias foram comparadas pelo teste de Duncan $(p \leq 0,05)$.

\section{Resultados e Discussão}

As sementes de ambos os híbridos apresentaram teor de água inicial entre $8,4 \%$ e 10,5\%. Após o teste de envelhecimento acelerado tradicional, esses valores alcançaram $29,0 \%$ a $31,5 \%$, para as sementes de SWB 551 e, $28,5 \%$ a $31,6 \%$, para as sementes de SWB 585, enquanto após o envelhecimento acelerado com uso de solução saturada de sal, os teores de água das sementes dos dois híbridos variaram de $11,2 \%$ a $12,7 \%$. Assim, essas variações estavam dentro de limites toleráveis, não afetando o comportamento das sementes durante a condução dos testes e contribuindo para a obtenção de resultados consistentes (Marcos Filho, 1999b; TeKrony, 2003).

Para os dois híbridos avaliados, em todas as épocas experimentais, as análises da variância dos dados obtidos indicaram efeitos significativos de lotes para a maioria dos testes conduzidos; os lotes apresentaram germinação entre $79 \%$ e $97 \%$ (Tabelas 1 e 2), superior ao limite mínimo (60\%) para a comercialização de sementes de milho superdoce (Brasil, 2005). O uso de lotes com poder germinativo semelhante é recomendável para que sejam comparados os desempenhos em testes de vigor, sendo importante, também, o uso de lotes que atendam as exigências estabelecidas para a comercialização (Powell, 1986; Marcos-Filho, 1999a). 
Na Tabela 1 são apresentados os resultados correspondentes ao híbrido SWB 551, nas três épocas de avaliação. Observou-se que, na primeira época experimental, os testes de germinação e envelhecimento acelerado tradicional detectaram o melhor desempenho do lote 5 e identificaram o pior desempenho do lote 3 , acompanhado pelo lote 4 no teste de germinação. Já o teste de envelhecimento acelerado com uso de solução saturada de sal destacou os lotes 1 e 4 como os mais vigorosos e o lote 2 como o menos vigoroso.

Tabela 1. Valores médios de germinação (G), envelhecimento acelerado tradicional (EAT), envelhecimento acelerado com solução saturada de $\mathrm{NaCl}$ (EASS), emergência de plântulas em campo (EPC) e índices de vigor (IV), uniformidade (Unif) e comprimento de plântulas (Comp) determinados em análises SVIS ${ }^{\circledR}$ de cinco lotes de sementes de milho superdoce SWB 551.

\begin{tabular}{|c|c|c|c|c|c|c|c|c|}
\hline \multirow{2}{*}{ Época } & \multirow{2}{*}{ Lote } & G & EAT & EASS & EPC & \multicolumn{3}{|c|}{ SVIS } \\
\hline & & \multicolumn{4}{|c|}{ 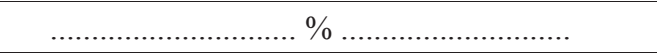 } & IV & Unif & Comp $(\mathrm{cm})$ \\
\hline \multirow{5}{*}{ Primeira } & 1 & $91 \mathrm{AB}$ & $79 \mathrm{AB}$ & $82 \mathrm{~A}$ & $84 \mathrm{~A}$ & $840 \mathrm{~A}$ & $912 \mathrm{~A}$ & $7,5 \mathrm{~A}$ \\
\hline & 2 & $91 \mathrm{AB}$ & $75 \mathrm{ABC}$ & $73 \mathrm{~B}$ & $82 \mathrm{~A}$ & $787 \mathrm{~A}$ & $905 \mathrm{~A}$ & $6,8 \mathrm{~A}$ \\
\hline & 3 & $86 \mathrm{~B}$ & $67 \mathrm{C}$ & $76 \mathrm{AB}$ & $77 \mathrm{~A}$ & $819 \mathrm{~A}$ & $925 \mathrm{~A}$ & $7,2 \mathrm{~A}$ \\
\hline & 4 & $86 \mathrm{~B}$ & $70 \mathrm{BC}$ & $81 \mathrm{~A}$ & $81 \mathrm{~A}$ & $866 \mathrm{~A}$ & $910 \mathrm{~A}$ & $7,9 \mathrm{~A}$ \\
\hline & 5 & $93 \mathrm{~A}$ & $80 \mathrm{~A}$ & $78 \mathrm{AB}$ & $78 \mathrm{~A}$ & $861 \mathrm{~A}$ & $910 \mathrm{~A}$ & $7,8 \mathrm{~A}$ \\
\hline \multicolumn{2}{|c|}{ CV (\%) } & 3,9 & 7,9 & 5,3 & 6,3 & 7,4 & 3,1 & 11,2 \\
\hline \multirow{5}{*}{ Segunda } & 1 & $91 \mathrm{~B}$ & $82 \mathrm{~A}$ & $80 \mathrm{~A}$ & $88 \mathrm{~A}$ & $872 \mathrm{~A}$ & $908 \mathrm{~A}$ & 7,9A \\
\hline & 2 & $90 \mathrm{~B}$ & $75 \mathrm{AB}$ & $79 \mathrm{~A}$ & $81 \mathrm{~A}$ & $753 \mathrm{~B}$ & $891 \mathrm{~A}$ & $6,4 \mathrm{~B}$ \\
\hline & 3 & $88 \mathrm{~B}$ & $71 \mathrm{~B}$ & $75 \mathrm{~A}$ & $83 \mathrm{~A}$ & $732 \mathrm{~B}$ & $928 \mathrm{~A}$ & $6,0 \mathrm{~B}$ \\
\hline & 4 & $89 \mathrm{~B}$ & $70 \mathrm{~B}$ & $73 \mathrm{~A}$ & $81 \mathrm{~A}$ & $733 B$ & $903 \mathrm{~A}$ & $6,1 \mathrm{~B}$ \\
\hline & 5 & $97 \mathrm{~A}$ & $80 \mathrm{~A}$ & $75 \mathrm{~A}$ & $89 \mathrm{~A}$ & $834 \mathrm{AB}$ & $913 \mathrm{~A}$ & $7,4 \mathrm{AB}$ \\
\hline \multicolumn{2}{|c|}{ CV (\%) } & 3,6 & 7,0 & 10,2 & 5,9 & 9,3 & 3,4 & 13,7 \\
\hline \multirow{5}{*}{ Terceira } & 1 & $87 \mathrm{~A}$ & $80 \mathrm{~A}$ & $79 \mathrm{~A}$ & $88 \mathrm{~A}$ & $797 \mathrm{~A}$ & $921 \mathrm{~A}$ & $6,9 \mathrm{~A}$ \\
\hline & 2 & $83 \mathrm{~A}$ & $65 \mathrm{BC}$ & $66 \mathrm{C}$ & $86 \mathrm{AB}$ & 691B & $905 \mathrm{~A}$ & $5,6 \mathrm{~B}$ \\
\hline & 3 & $82 \mathrm{~A}$ & $61 \mathrm{C}$ & $72 B$ & $84 \mathrm{AB}$ & $672 B$ & $922 \mathrm{~A}$ & $5,2 \mathrm{~B}$ \\
\hline & 4 & $86 \mathrm{~A}$ & $59 \mathrm{C}$ & $60 \mathrm{D}$ & $79 \mathrm{~B}$ & $664 \mathrm{~B}$ & $935 \mathrm{~A}$ & $5,1 \mathrm{~B}$ \\
\hline & 5 & $87 \mathrm{~A}$ & $72 \mathrm{~B}$ & $76 \mathrm{AB}$ & $84 \mathrm{AB}$ & $671 \mathrm{~B}$ & $900 \mathrm{~A}$ & $5,3 \mathrm{~B}$ \\
\hline \multicolumn{2}{|c|}{ CV (\%) } & 6,3 & 7,1 & 4,8 & 5,9 & 7,4 & 2,6 & 11,7 \\
\hline
\end{tabular}

Médias seguidas da mesma letra (dentro de cada coluna) não diferem entre si pelo teste de Duncan, $\mathrm{p} \leq 0,05$.

Por outro lado, embora não tenha detectado diferenças significativas entre os lotes, a análise computadorizada SVIS ${ }^{\circledR}$ apresentou resultados semelhantes aos obtidos no teste de emergência de plântulas em campo. Essa relação é importante, uma vez que a emergência de plântulas constitui-se parâmetro indicador da eficiência dos testes para avaliação do potencial fisiológico dos lotes de sementes (Marcos-Filho, 1999a).

Já na segunda época de avaliação, os parâmetros índice de vigor e comprimento de plântulas da análise SVIS $^{\circledR}$ permitiram a identificação dos lotes mais (lote 1) e menos vigorosos (lotes 2,3 e 4 ); o SVIS ${ }^{\circledR}$, juntamente com o teste de envelhecimento acelerado tradicional, foram os únicos procedimentos que classificaram os lotes em diferentes níveis de vigor, apresentando resultados semelhantes entre si. Vale ressaltar que, em relação ao teste de envelhecimento acelerado tradicional, as informações proporcionadas nessa época foram coerentes com as obtidas na época anterior, sendo o lote 5 identificado novamente como o de melhor desempenho, porém, acompanhado pelo lote 1, e os piores desempenhos foram apresentados pelos lotes 3 e 4 . Há relatos na literatura constando indicações de sucesso do teste de envelhecimento acelerado tradicional na avaliação do vigor de sementes de milho doce (Wilson Junior et al., 1992; Hampton e TeKrony, 1995; Santos et al., 2002).

$\mathrm{Na}$ terceira época experimental, os dados de todos os testes de vigor apontaram diferenças significativas entre os lotes, destacando a superioridade do lote 1. Quanto 
ao pior desempenho, o lote 4 se destacou nos testes de envelhecimento acelerado com uso de solução saturada de sal e emergência de plântulas em campo, sendo acompanhado pelo lote 3 no teste de envelhecimento acelerado tradicional, e pelos lotes 2, 3 e 5 na análise SVIS ${ }^{\circledR}$ (índice de vigor e comprimento de plântulas). Assim, confirmou-se a sensibilidade do sistema automatizado na detecção de diferenças de vigor, observada na segunda época experimental. Cabe salientar que, embora o SVIS ${ }^{\circledR}$ não tenha estratificado os lotes quanto ao vigor de forma análoga aos demais testes, verificou-se que os resultados obtidos foram comparáveis entre si. A eficiência da análise computadorizada SVIS ${ }^{\circledR}$, em comparação com procedimentos tradicionais, também foi constatada na avaliação do potencial fisiológico de sementes de melão (Marcos-Filho et al., 2006), feijão (Gomes Junior e Chamma, 2008) e soja (Marcos-Filho et al., 2009).

$\mathrm{Na}$ Tabela 2 são apresentados os resultados referentes às determinações efetuadas com sementes do híbrido SWB
585. Diferindo do híbrido SWB 551, a análise $\mathrm{SVIS}^{\circledR}$, no que diz respeito ao índice de vigor e ao comprimento de plântulas, detectou diferenças significativas entre lotes na primeira época de avaliação, sendo o lote 9 destacado como o menos vigoroso. De forma coerente, o teste de envelhecimento acelerado tradicional também apontou o lote 9 como o de pior desempenho. Como as diferenças entre o potencial fisiológico das sementes desse híbrido eram mais estreitas, houve dificuldade para a identificação do lote mais vigoroso, sendo apenas o teste de envelhecimento acelerado tradicional sensível para detectar a superioridade do lote 6. É importante salientar que as sementes de milho superdoce naturalmente possuem baixo potencial fisiológico como consequência de uma série de características físicoquímicas e fisiológicas intrínsecas desta espécie, destacadas na literatura (Tosello, 1987; Chern e Sung, 1991; Douglas et al., 1993; Silva, 1994). É muito provável que isto tenha interferido nos resultados, dificultando uma separação mais consistente dos lotes quanto ao vigor.

Tabela 2. Valores médios de germinação (G), envelhecimento acelerado tradicional (EAT), envelhecimento acelerado com solução saturada de $\mathrm{NaCl}$ (EASS), emergência de plântulas em campo (EPC) e índices de vigor (IV), uniformidade (Unif) e comprimento de plântulas (Comp) determinados em análises SVIS ${ }^{\circledR}$ de cinco lotes de sementes de milho superdoce SWB 585.

\begin{tabular}{|c|c|c|c|c|c|c|c|c|}
\hline \multirow{2}{*}{ Época } & \multirow{2}{*}{ Lote } & G & EAT & EASS & EPC & \multicolumn{3}{|c|}{ SVIS } \\
\hline & & \multicolumn{4}{|c|}{ 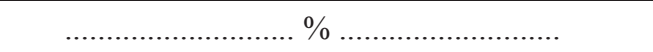 } & IV & Unif & Comp (cm) \\
\hline \multirow{5}{*}{ Primeira } & 6 & $89 \mathrm{~A}$ & $69 \mathrm{~A}$ & $72 \mathrm{~A}$ & $82 \mathrm{~A}$ & $836 \mathrm{~A}$ & $895 \mathrm{~A}$ & $7,9 \mathrm{~A}$ \\
\hline & 7 & $90 \mathrm{~A}$ & $51 \mathrm{~B}$ & $74 \mathrm{~A}$ & $76 \mathrm{~A}$ & $905 \mathrm{~A}$ & $905 \mathrm{~A}$ & $8,9 \mathrm{~A}$ \\
\hline & 8 & $91 \mathrm{~A}$ & $53 \mathrm{~B}$ & $61 \mathrm{~B}$ & $74 \mathrm{~A}$ & $732 \mathrm{~B}$ & $889 \mathrm{~A}$ & $6,5 \mathrm{~B}$ \\
\hline & 9 & $88 \mathrm{~A}$ & $33 \mathrm{C}$ & $70 \mathrm{~A}$ & $74 \mathrm{~A}$ & $655 \mathrm{C}$ & $890 \mathrm{~A}$ & $5,4 \mathrm{C}$ \\
\hline & 10 & $90 \mathrm{~A}$ & $61 \mathrm{AB}$ & $77 \mathrm{~A}$ & $84 \mathrm{~A}$ & $897 \mathrm{~A}$ & $896 \mathrm{~A}$ & $8,8 \mathrm{~A}$ \\
\hline \multicolumn{2}{|c|}{ CV $(\%)$} & 5,0 & 18,2 & 8,5 & 8,9 & 6,1 & 1,9 & 9,2 \\
\hline \multirow{5}{*}{ Terceira } & 6 & $83 \mathrm{AB}$ & $65 \mathrm{~A}$ & $63 \mathrm{~A}$ & $84 \mathrm{AB}$ & $642 \mathrm{~A}$ & $854 \mathrm{~B}$ & $5,4 \mathrm{~A}$ \\
\hline & 7 & $89 \mathrm{~A}$ & $50 \mathrm{BC}$ & $66 \mathrm{~A}$ & $86 \mathrm{~A}$ & $619 \mathrm{AB}$ & $886 \mathrm{AB}$ & $5,0 \mathrm{AB}$ \\
\hline & 8 & $87 \mathrm{~A}$ & $45 \mathrm{CD}$ & $62 \mathrm{~A}$ & $81 \mathrm{AB}$ & $558 \mathrm{~B}$ & $894 \mathrm{AB}$ & $4,1 \mathrm{~B}$ \\
\hline & 9 & 79B & $38 \mathrm{D}$ & $53 \mathrm{~B}$ & $78 \mathrm{~B}$ & $564 \mathrm{~B}$ & $868 \mathrm{AB}$ & $4,3 \mathrm{~B}$ \\
\hline & 10 & $86 \mathrm{AB}$ & $53 \mathrm{~B}$ & $67 \mathrm{~A}$ & $82 \mathrm{AB}$ & $562 \mathrm{~B}$ & $902 \mathrm{~A}$ & 4,1B \\
\hline \multicolumn{2}{|c|}{ CV (\%) } & 5,7 & 10,3 & 8,7 & 5,8 & 7,3 & 3,2 & 13,5 \\
\hline
\end{tabular}

Médias seguidas da mesma letra (dentro de cada coluna) não diferem entre si pelo teste de Duncan, $\mathrm{p} \leq 0,05$.

Os resultados dos testes realizados na terceira época experimental (Tabela 2), confirmaram o pior desempenho do lote 9 , acompanhado pelos lotes 8 e 10 nos parâmetros índice de vigor e comprimento de plântulas da análise SVIS $^{\circledR}$. Esses parâmetros também permitiram a identificação do lote mais vigoroso (lote 6), concordando com a informação obtida no teste de envelhecimento acelerado tradicional. Avaliando o vigor de sementes de soja, Marcos-Filho et al. (2009) verificaram resultados coerentes entre a análise computadorizada SVIS $^{\circledR}$ e o teste de envelhecimento acelerado.

Diante dos resultados obtidos para o híbrido SWB 585 (Tabela 2), verificou-se que, dentre todos os testes de vigor 
conduzidos, o teste de envelhecimento acelerado tradicional foi o único que classificou os lotes em diferentes níveis de vigor de maneira semelhante, na primeira e na segunda época de avaliação. Embora haja vários procedimentos disponíveis para a determinação do vigor de sementes, o teste de envelhecimento acelerado tem sido um dos mais utilizados (McDonald, 1995), proporcionando informações com alto grau de consistência (TeKrony, 1995). Para ambos os híbridos avaliados nesta pesquisa, esse teste apresentou eficiência na classificação dos lotes quanto ao vigor. Assim, pode ser constatada a sensibilidade da análise computadorizada SVIS ${ }^{\circledR}$ para a determinação do vigor de sementes de milho superdoce, uma vez que seus resultados foram coerentes com os obtidos no teste de envelhecimento acelerado tradicional.

\section{Conclusões}

A análise computadorizada de imagens de plântulas SVIS $^{\circledR}$ é eficiente para a determinação do vigor de sementes de milho superdoce, com sensibilidade comparável ao teste de envelhecimento acelerado tradicional.

\section{Agradecimentos}

À FAPESP e ao CNPq pela concessão das bolsas de estudo e recursos para condução da pesquisa e à empresa Dow AgroSciences pela cessão das sementes.

\section{Referências}

BRASIL. Ministério da Agricultura, Pecuária e Abastecimento. Padrões para produção e comercialização de sementes de milho - cultivares híbridas. Instrução normativa n.25, de 16 de dezembro de 2005. Diário Oficial, Brasília, sec.1, n.243, p.18 de 20/12/2005, 2005.

BRASIL. Ministério da Agricultura, Pecuária e Abastecimento. Regras para análise de sementes. Ministério da Agricultura, Pecuária e Abastecimento. Secretaria de Defesa Agropecuária. Brasília, DF: MAPA/ACS, 2009. 395p. http://www.agricultura.gov.br/arq_editor/file/laborat $\% \mathrm{C} 3 \% \mathrm{~B} 3$ rio/ sementes/regras $\% 20$ para $\% 20$ analise $\% 20$ de $\% 20$ sementes.pdf

CHERN, G.S.; SUNG, F.J.M. Prevention of injury during imbibition in shrunken-2 corn seeds by osmotic control of water uptake. Seed Science and Technology, v.19, n.3, p.469-476, 1991.

DOUGLAS, S.K.; JUVIK, J.A.; SPLITTSTOESSER, W.E. Sweet corn seedling emergence and variation in kernel carbohydrate reserves. Seed Science and Technology, v.21, n.3, p.433-445, 1993.

GOMES JUNIOR, F.G.; CHAMMA, H.M.C.P. Eficiência de um sistema computadorizado de processamento de imagens para avaliação do vigor de sementes de feijão. In: CONGRESSO NACIONAL DE PESQUISA DE FEIJÃO, 2008, Campinas. Anais... Campinas: Instituto Agronômico de Campinas, p.685-688, 2008.

GOMES JUNIOR, F.G.; MONDO, V.H.V.; CICERO, S.M.; McDONALD, M.B.; BENNETT, M.A. Evaluation of priming effects on sweet corn seeds by SVIS. Seed Technology, v.31, n.1, p.95-100, 2009.

GUISCEM, J.M.; NAKAGAWA, J.; ZUCARELI, C. Qualidade fisiológica de sementes de milho doce BR 400 (BT) em função do teor de água na colheita e da temperatura de secagem. Revista Brasileira de Sementes, v.24, n.1, p.220-228, 2002. http://www.scielo.br/pdf/rbs/ v24n1/v24n1a31.pdf

HAMPTON, J.G.; TEKRONY, D.M. Handbook of vigour test methods. 3.ed. Zurich: ISTA, 1995. 117p.

HOFFMASTER, A.L.; FUJIMURA, K.; McDONALD, M.B.; BENNETT, M.A. An automated system for vigor testing three-day-old soybean seedlings. Seed Science and Technology, v.31, n.3, p.701-713, 2003. http:// docserver.ingentaconnect.com/deliver/connect/ista/02510952/v31n3/ s19.pdf? expires $=1317671172 \& \mathrm{id}=64700774 \&$ titleid $=75001181 \&$ accna me $=$ universidade + sao + paulo + if $\&$ checksum $=552348 \mathrm{a} 5 \mathrm{cc} 24 \mathrm{afef} 66 \mathrm{c} 80 \mathrm{ab}$ 156a49444

HOFFMASTER, A.F.; XU, L.; FUJIMURA, K.; McDONALD, M.B.; BENNETT, M.A.; EVANS, A.F. The Ohio State University Seed Vigor Imaging System (SVIS) for soybean and corn seedlings. Seed Technology, v.27, n.1, p.7-24, 2005.

JIANHUA, Z.; McDONALD, M.B. The saturated salt accelerated aging test for small-seeded crops. Seed Science and Technology, v.25, n.1, p.123-131, 1996.

MARCOS-FILHO, J. Testes de envelhecimento acelerado. In: KRZYZANOWSKI, F.C.; VIEIRA, R.D.; FRANÇA-NETO, J.B. (Ed.). Vigor de sementes: conceitos e testes. Londrina: ABRATES, cap.3, p.1-24, 1999b.

MARCOS-FILHO, J. Testes de vigor: importância e utilização. In: KRZYZANOWSKI, F.C.; VIEIRA, R.D.; FRANÇA-NETO, J.B. (Ed.). Vigor de sementes: conceitos e testes. Londrina: ABRATES, cap.1, p.1-21, 1999a.

MARCOS-FILHO, J.; BENNETT, M.A.; McDONALD, M.B.; EVANS, A.F.; GRASSBAUGH, E.M. Assessment of melon seed vigour by an automated computer imaging system compared to traditional procedures. Seed Science and Technology, v.34, n.2, p.485-497, 2006. http://docserver. ingentaconnect.com/deliver/connect/ista/02510952/v34n2/s23.expires= $1317671715 \&$ id $=64700967 \&$ titleid $=75001181 \&$ accname $=$ universidade + sao + paulo + if $\&$ checksum $=$ d0758cf5ca4633ecf7700169acef $9 \mathrm{bcf}$

MARCOS-FILHO, J.; KIKUTI, A.L.P.; LIMA, L.B. Métodos para avaliação do vigor de sementes de soja, incluindo a análise computadorizada de imagens. Revista Brasileira de Sementes, v.31, n.1, p.102-112, 2009. http://www.scielo.br/pdf/rbs/v31n1/a12v31n1.pdf

McCORMAC, A.C.; KEEFE, P.D.; DRAPER, R.S. Automated vigour testing of field vegetables using image analysis. Seed Science and 
Technology, v.18, n.1, p.103-112, 1990.

McDONALD, M.B. Standardization of seed vigour tests. In: CONGRESS OF THE INTERNATIONAL SEED TESTING ASSOCIATION, 24., 1995, Copenhagen, Denmark. Proceedings... Zürich, CH-Switzerland: ISTA, 1995. p.88-97.

OTONI, R.R.; McDONALD, M.B. Moisture and temperature effects on maize and soybean seedlings using the seed vigor imaging system. Seed Technology, v.27, n.2, p.243-247, 2005.

POWELL, A.A. Cell membranes and seed leachate conductivity in relation to the quality of seed for sowing. Journal of Seed Technology, v.10, n.2, p.81-100, 1986.

RAMOS, N.P.; MARCOS FILHO, J.; GALLI, J.A. Tratamento fungicida em semente de milho super-doce. Revista Brasileira de Sementes, v.30, n.1, p.24-31, 2008. http://www.scielo.br/pdf/rbs/v30n1/a04v30n1.pdf

SAKO, Y.; McDONALD, M.B.; FUJIMURA, K.; EVANS, A.F.; BENNETT, M.A. A system for automated seed vigour assessment. Seed Science and Technology, v.29, n.3, p.625-636, 2001.

SANTOS, P.M.; GONDIM, T.C.O.; ARAÚJO, E.F.; DIAS, D.C.F.S. Avaliação da qualidade fisiológica de sementes de milho-doce pelo teste de envelhecimento acelerado. Revista Brasileira de Sementes, v.24, n.1, p.91-96, 2002. http://www.scielo.br/pdf/rbs/v24n1/v24n1a13.pdf

SAS INSTITUTE. Statistical Analysis System: getting stared with the SAS learning. Cary, 2000. 81p.

SILVA, N. Melhoramento de milho doce. In: ENCONTRO SOBRE TEMAS DE GENÉTICA E MELHORAMENTO, 11., 1994, Piracicaba. Anais... Piracicaba: ESALQ, 1994. v.11, p.45-49.
STYER, R.C.; CANTLIFFE, D.J. Dependence of seed vigor during germination on carbohydrate source in endosperm mutants of maize. Plant Physiology, v.76, n.1, p.196-200, 1984. http://www.plantphysiol. org/content $/ 76 / 1 / 196$. full.pdf + html? sid $=4 \mathrm{fb} 1 \mathrm{c} 2 \mathrm{e} 2-\mathrm{e} 9 \mathrm{cb}-4 \mathrm{a} 30-8983$ 2a0bcd6f 4973

STYER, R.C.; CANTLIFFE, D.J.; HANNAH, L.C. Differential seed and seedling vigor in Shrunken-2 compared to three other genotypes of corn at various stages of development. Journal of the American Society for Horticultural Science, v.105, n.3, p.329-332, 1980.

TEKRONY, D.M. Accelerated aging. In: VAN DE VENTER, H.A. (Ed.). Seed vigour testing seminar. Copenhagen: ISTA, 1995. p.53-72.

TEKRONY, D.M. Precision is an essential component in seed vigour testing. Seed Science and Technology, v.31, n.2, p.435-447, 2003. http:// docserver.ingentaconnect.com/deliver/connect/ista/02510952/v31n2/ s20.pdf?expires $=1317671861 \& \mathrm{id}=64701011 \&$ titleid $=75001181 \&$ accna me $=$ universidade + sao + paulo + if $\&$ checksum $=$ a 7 f2acc38b9e $331822 \mathrm{ed} 58$ $5 \mathrm{c} 54 \mathrm{a} 18573$

TOSELLO, G.A. Milhos especiais e seu valor nutritivo. In: PATERNIANI, E.; VIEGAS, G.P. (Eds.) Melhoramento e produção de milho. Campinas: Fundação Cargill. v.1, p.375-409, 1987.

WANN, E.V. Seed vigor and respiration of maize kernels with different endosperm genotypes. Journal of the American Society for Horticultural Science, v.105, n.1, p.31-34, 1980.

WILSON JUNIOR, D.O.; ALLEYNE, J.C.; SHAFII, B.; MOHAN, S.K. Combining vigour test results for prediction of final stand of shrunken-2 sweet corn seed. Crop Science, v.32, n.6, p.1496-1502, 1992. 